\title{
Numerical Method for 2D Quasi-linear Hyperbolic Equation on an Irrational Domain: Application to Telegraphic Equation
}

\author{
Bishnu Pada Ghosh ${ }^{1}$ and Nepal Chandra Roy ${ }^{2 *}$ \\ ${ }^{1}$ Department of Mathematics, Jagannath University, Dhaka 1100, Bangladesh \\ ${ }^{2}$ Department of Mathematics, Dhaka University, Dhaka 1000, Bangladesh
}

(Received : 6 September 2021; Accepted : 14 October 2021)

\begin{abstract}
We develop a novel three-level compact method (implicit) of second order in time and space directions using unequal grid for the numerical solution of 2D quasi-linear hyperbolic partial differential equations on an irrational domain. The stability analysis of the model problem for unequal mesh is discussed and it is revealed that the developed scheme is unconditionally stable for the Telegraphic equation. For linear difference equations on an irrational domain, the alternating direction implicit method is discussed. The projected technique is scrutinized on several physical problems on an irrational domain to exhibitthe accuracy and effectiveness of the suggested method.
\end{abstract}

Keywords: Quasi-linear; Unequal mesh; Irrational domain; Telegraphic equation; Van der Pol equation; Dissipative equation.

\section{Introduction}

Partial differential equations (PDEs) have a variety of applications in the field of engineering and physical sciences. The hyperbolic PDEs model the vibration of structure (e.g., beams, buildings, and machines) and are the basis for fundamental equations of atomic physics. Linear hyperbolic PDEs elucidate the linear phenomena which arise in numerous areas such as biology, industry, aerospace, etc., whereas nonlinear phenomena take place in various areas (e.g., fluid dynamics, mathematical biology, solid-state physics, plasma physics, and chemical kinematics), which can be described by nonlinear PDEs. The approximate solution of 2D second-order quasi-linear hyperbolic PDEs plays a vital role in numerous areas such as engineering, mathematical and physical sciences.

Let us consider the 2D quasi-linear hyperbolic PDEs

$$
\begin{aligned}
\phi_{t t} & =A(x, y, t, \phi) \phi_{x x}+B(x, y, t, \phi) \phi_{y y} \\
& +R\left(x, y, t, \phi, \phi_{x}, \phi_{y}, \phi_{t}\right) \\
& 0<x<a, 0<y<b, t>0
\end{aligned}
$$

with two initial conditions prescribed by

$$
\begin{aligned}
& \phi(x, y, 0)=a_{0}(x, y), \quad \phi_{t}(x, y, 0)=a_{1}(x, y), \\
& 0 \leq x \leq a, 0 \leq y \leq b,
\end{aligned}
$$

and prescribed boundary conditions are

$$
\begin{aligned}
& \phi(0, y, t)=b_{0}(y, t), \quad \phi(a, y, t)=b_{1}(y, t), \\
& 0 \leq y \leq b, t>0, \\
& \phi(x, 0, t)=c_{0}(x, t), \quad \phi(x, b, t)=c_{1}(x, t), \\
& 0 \leq x \leq a, t>0 .
\end{aligned}
$$

Here $A(x, y, t, \phi)>0, B(x, y, t, \phi)>0$ and $A, B, \phi$, $a_{0}, a_{1}$ are satisfactorily regular, and also their required higher order partial derivatives are defined analytically in the solution region $\Omega \equiv\{(x, y, t): 0<x<a, 0<y<b$, $t>0\}$. The initial and boundary conditions (2)-(3) are

*Author for correspondence. e-mail: nepal@du.ac.bd given with required regularity to remain the order of the method unchanged. Besides we presume that there exists exactly one regular solution for the initial boundary value problem (IBVP) (1)-(3). Required information is discussed by Li et al ${ }^{1}$.

It has been experienced in the past that the nonlinear partial differential equations are more complicated to solve analytically and there are no general methods exist for the solution of such equations. Therefore, stable numerical methods are the only choice to handle such problems. The existence and uniqueness of the solution of 1D nonlinear $2^{\text {nd }}$ order hyperbolic PDEs have been discussed by $\mathrm{Li}$ et al. ${ }^{1}$.In 1968, Greenspan ${ }^{2}$ has introduced a boundary value technique to obtain an approximate solution of the wave equation. Ciment and Leventhal ${ }^{3,4}$ have studied the fourthorder compact implicit method to solve wave equations. Any explicit scheme for the second order hyperbolic equation is stable for a certain stability range. However, Twizell $^{5}$ has established a new compact scheme for the wave equation with an improved stability range. Mohanty et al. ${ }^{6}$ have discussed a higher order numerical scheme for the solution of the 2D second order nonlinear hyperbolic PDEs. Further, Mohanty et al. ${ }^{7}$ have developed the method to approximate the solution of second order quasi-linear hyperbolic PDEs. All the developed schemes ${ }^{5-7}$ are not unconditionally stable. Many researchers ${ }^{8-17}$ have proposed implicit methods for the solution of 2D linear hyperbolic PDEs. All numerical methods discussed in $^{1-17}$ are based on equal mesh discretization and not applicable to find the approximate solution if the ratio of the sides of the solution domain is an irrational number. Unequal mesh discretization plays an important role when the solution domain is irrational. Most recently, Priyadarshini and Mohanty ${ }^{18,19}$ have presented novel ideas to approximate the solution of 2D quasi-linear elliptic PDEs on an irrational domain.

To the authors' knowledge, no high precision numerical scheme using unequal mesh for the solution of 2D quasilinear hyperbolic PDEs on an irrational domain has been 
discussed in the literature so far. The mesh sizes cannot be equal in both $x$ - and $y$ - coordinates in the case of an irrational domain. Therefore, the development of a suitable high precision approximate solution using different mesh sizes for the hyperbolic PDE (1) plays a significant role. In the present work, we discuss a novel three-level compact implicit scheme of order of accuracy two in time and space directions for the solution of 2D quasi-linear hyperbolic PDE (1) on an irrational domain. The article is displayed as follows: In section II, the scheme has been formulated based on an unequal mesh and provides a total derivation of the numerical scheme. In section III, we discuss the stability analysis and alternative direction implicit (ADI) method for 2D Telegraphic equation. In section IV, several hyperbolic equations of physical repute have been computed to clarify and scrutinize the accuracy of the recommended methods. Finally, section V summarizes all the steps.

\section{Conceptualization and Derivation of the Numerical Method}

Now, we consider the 2D nonlinear hyperbolic PDE of the form

$\phi_{t t}=A(x, y, t) \phi_{x x}+B(x, y, t) \phi_{y y}$

$+R\left(x, y, t, \phi, \phi_{x}, \phi_{y}, \phi_{t}\right)$,

$0<x<a, 0<y<b, t>0$

where $a / b$ is an irrational number, $A(x, y, t)>0, B(x, y, t)>0$ and equations (1)-(4) represent the initial and boundary conditions. Let the grid spacing in $x$-, $y$ - and $t$-coordinates be $h_{x}>0, h_{y}>0$ and $k>0$, respectively. The domain $\Omega \equiv\{(x, y, t): 0<x<a, 0<y<b, t>0\}$ is sheathed by a set of mesh points $\left(x_{l}, y_{m}, t_{j}\right)$, where $x_{l}=l h_{x}, l=$ $0(1) N+1 ; y_{m}=m h_{y}, m=1(1) M+1 ; \quad \& t_{j}=j k, 0<$ $j<J$, where $N, M, \& J$ are positive integersand $(N+1) h_{x}=$ $a,(M+1) h_{y}=b$. Let us defined the mesh ratio parameters by $P_{x}=\frac{k}{h_{x}}>0$ and $P_{y}=\frac{k}{h_{y}}>0$.

Let $\Phi_{l, m}^{j}=\phi\left(x_{l}, y_{m}, t_{j}\right)$ be the exact solution value of $\phi(x, y, t)$ at the nodal point $\left(x_{l}, y_{m}, t_{j}\right)$, and $\phi_{l, m}^{j}$ approximates $\Phi_{l, m}^{j}$. Let $A_{l, m}^{j}=A\left(x_{l}, y_{m}, t_{j}\right), B_{l, m}^{j}=$ $B\left(x_{l}, y_{m}, t_{j}\right), A_{x_{l, m}}^{j}=A_{x}\left(x_{l}, y_{m}, t_{j}\right), \quad B_{x_{l, m}}^{j}=$ $B_{x}\left(x_{l}, y_{m}, t_{j}\right), \ldots$, be the exact values of $A(x, y, t), B(x, y, t), A_{x}(x, y, t), B_{x}(x, y, t), \ldots . . e t c$. at the nodal point $\left(x_{l}, y_{m}, t_{j}\right)$, respectively.

At the mesh point $\left(x_{l}, y_{m}, t_{j}\right)$, we may write the nonlinear differential equation (5) as

$\Phi_{t t_{l, m}^{j}}^{j}-A_{l, m}^{j} \Phi_{x x_{l, m}^{j}}^{j}-B_{l, m}^{j} \Phi_{y y_{l, m}^{j}}^{j}=$

$R\left(x_{l}, y_{m}, t_{j}, \Phi_{l, m}^{j}, \Phi_{x_{l, m}}^{j}, \Phi_{y_{l, m}}^{j}, \Phi_{t}^{j}\right) \equiv R_{l, m}^{j}$

At the grid points $\left(x_{l}, y_{m}, t_{j}\right)$, we require the following approximations:

$$
\begin{aligned}
& \Phi_{t_{l, m}^{j}}^{j}=\frac{1}{2 k}\left[\Phi_{l, m}^{j+1}-\Phi_{l, m}^{j-1}\right]=\Phi_{t_{l, m}}^{j}+O\left(k^{2}\right), \\
& \Phi_{x_{l, m}^{j}}^{j}=\frac{1}{2 h_{x}}\left[\Phi_{l+1, m}^{j}-\Phi_{l-1, m}^{j}\right] \\
& =\Phi_{x l, m}^{j}+O\left(h_{x}^{2}\right), \\
& \bar{\Phi}_{y_{l, m}^{j}}^{j}=\frac{1}{2 h_{y}}\left[\Phi_{l, m+1}^{j}-\Phi_{l, m-1}^{j}\right] \\
& =\Phi_{y_{l, m}^{j}}^{j}+O\left(h_{y}^{2}\right), \\
& =\Phi_{x x_{l, m}^{j}}^{j}+O\left(h_{x}^{2}\right), \\
& \bar{\Phi}_{y y_{l, m}^{j}}^{j}=\frac{1}{h_{x}^{2}}\left[\Phi_{l, m+1}^{j}-2 \Phi_{l, m}^{j}+\Phi_{l, m-1}^{j}\right] \\
& =\Phi_{y y_{l, m}^{j}}^{j}+O\left(h_{y}^{2}\right) \\
& \bar{\Phi}_{t t_{l, m}^{j}}^{j}=\frac{1}{k^{2}}\left[\Phi_{l, m}^{j+1}-2 \Phi_{l, m}^{j}+\Phi_{l, m}^{j-1}\right] \\
& =\Phi_{t t_{l, m}^{j}}^{j}+O\left(k^{2}\right),
\end{aligned}
$$

We require the following approximation for $R\left(x, y, t, \phi, \phi_{x}, \phi_{y}, \phi_{t}\right)$.

$\bar{R}_{l, m}^{j}=R\left(x_{l}, \mathrm{y}_{\mathrm{m}}, t_{j}, \Phi_{l, m}^{j}, \bar{\Phi}_{x_{l, m}}^{j}, \bar{\Phi}_{y_{l, m}}^{j}, \bar{\Phi}_{t_{l, m}}^{j}\right)$

$=R_{l, m}^{j}+O\left(k^{2}+h_{x}^{2}+h_{y}^{2}\right)$,

With the aim to derive finite difference scheme for the HPDE (5), consider the following linear combination:

$$
\begin{aligned}
& R_{l, m}^{j}+P_{1} h_{x} R_{x_{l, m}}^{j}+P_{2} h_{y} R_{y_{l, m}}^{j}+P_{3} k R_{t l, m}^{j} \\
& =\bar{\Phi}_{t t_{l, m}^{j}}^{j}-h_{x} P_{1} A_{l, m}^{j} \Phi_{x x x_{l, m}}^{j}-h_{y} P_{2} B_{l, m}^{j} \Phi_{y y y_{l, m}}^{j} \\
& -\left[A_{l, m}^{j}+h_{x} P_{1} A_{x l, m}^{j}+h_{y} P_{2} A_{y_{l, m}^{j}}^{j}+P_{3} k A_{t l, m}^{j}\right] \bar{\Phi}_{x x_{l, m}^{j}}^{j} \\
& -\left[B_{l, m}^{j}+h_{x} P_{1} B_{x_{l, m}}^{j}+h_{y} P_{2} B_{y_{l, m}}^{j}+P_{3} k B_{t l, m}^{j}\right] \bar{\Phi}_{y y_{l, m}^{j}}^{j} \\
& +P_{3} k \Phi_{t t t_{l, m}}^{j}-P_{3} k A_{l, m}^{j} \Phi_{x x t_{l, m}}^{j}-P_{3} k B_{l, m}^{j} \Phi_{y y t_{l, m}}^{j} \\
& -h_{x} P_{2} A_{l, m}^{j} \Phi_{x x y_{l, m}}^{j}-h_{y} P_{1} B_{l, m}^{j} \Phi_{x y y_{l, m}}^{j} \\
& +O\left(k^{2}+h_{x}^{2}+h_{y}^{2}\right)
\end{aligned}
$$

Equating the coefficients of $\Phi_{x x x_{l, m}}^{j}, \Phi_{y y y_{l, m}}^{j}$ and $\Phi_{t t t_{l, m}}^{j}$ to zero, we get

$$
P_{1}=0, P_{2}=0, \quad P_{3}=0 \text {. }
$$

Thus,

$$
\begin{gathered}
L_{\phi} \equiv \bar{\Phi}_{t t_{l, m}^{j}}^{j}-A_{l, m}^{j} \bar{\Phi}_{x x_{l, m}^{j}}^{j}-B_{l, m}^{j} \bar{\Phi}_{y y_{l, m}^{j}}^{j} \\
=\bar{R}_{l, m}^{j}+O\left(k^{2}+h_{x}^{2}+h_{y}^{2}\right) .
\end{gathered}
$$


Then the approximation for the PDEs (5) at each nodal point $\left(x_{l}, y_{m}, t_{j}\right)$ is given by

$$
\begin{gathered}
L_{\phi} \equiv \bar{\Phi}_{t t_{l, m}^{j}}^{j}-A_{l, m}^{j} \bar{\Phi}_{x x_{l, m}^{j}}^{j}-B_{l, m}^{j} \bar{\Phi}_{y y_{l, m}^{j}}^{j} \\
=\bar{R}_{l, m}^{j}+\bar{T}_{l, m}^{j}
\end{gathered}
$$

for $l=1$ (1) $N, m=1$ (1) $M, j=0,1,2, \ldots J$,

where the local truncation error (LTE),

$$
\bar{T}_{l, m}^{j}=O\left(k^{2}+h_{x}^{2}+h_{y}^{2}\right)
$$

Finally, using the approximations (10)-(13) from (15) and (16), the LTE is obtained as

$\bar{T}_{l, m}^{j}=O\left(k^{2}+h_{x}^{2}+h_{y}^{2}\right)$.

Thus, the order of the proposed method is $O\left(k^{2}+h_{x}^{2}+\right.$ $\left.h_{y}^{2}\right)$.

Incorporating the prescribed conditions (2)-(4), the method (16) can be expressed in a block tri-diagonal matrix form at each advanced time level. We use the operator splitting method or ADI method for linear PDEs, and NewtonRaphson for nonlinear or quasi-linear PDEs ${ }^{20,21}$.

\section{Stability Analysis and Operator Splitting Technique}

The mathematical modelling of well-known linear telegraphic equation in two-space dimension along with forcing function is given by

$\phi_{t t}+2 \alpha_{0} \phi_{t}+\beta_{0}^{2} \phi=\phi_{x x}+\phi_{y y}+f(x, y, t)$,

$\alpha_{0}>0, \beta_{0} \geq 0$,

subject to prescribed initial and Dirichlet boundary conditions stated in (2)-(4). Here $\alpha_{0}, \beta_{0}$ are constants. Equation (18) represents a damped wave equation when $\beta_{0}=0$. In this section, we denote $a=\alpha_{0}{ }^{2} k^{2}, b=$ $\beta_{0}{ }^{2} k^{2}, P_{x}=\frac{k}{h_{x}}>0$ and $P_{y}=\frac{k}{h_{y}}>0$.

Applying the scheme (16) to the PDE (18), neglecting error terms, we get

$$
\begin{aligned}
& {\left[\delta_{t}^{2}-\sqrt{a}\left(2 \mu_{t} \delta_{t}\right)+b-P_{x}^{2} \delta_{x}^{2}-P_{y}^{2} \delta_{y}^{2}\right] \phi_{l, m}^{j}} \\
& =k^{2} f_{l, m}^{j} .
\end{aligned}
$$

where $\quad \delta_{x} \phi_{l, m}^{j}=\phi_{l+\frac{1}{2}, m}^{j}-\phi_{l-\frac{1}{2}, m}^{j} \quad$ and $\quad \mu_{x} \phi_{l, m}^{j}=$ $\frac{1}{2}\left[\phi_{l+\frac{1}{2}, m}^{j}+\phi_{l-\frac{1}{2}, m}^{j}\right]$ are central and average difference approximations in the $x$-direction, etc and $f_{l, m}^{j}=$ $f\left(x_{l}, y_{m}, t_{j}\right)$.

The above linear scheme is not unconditionally stable ${ }^{7}$. To find an unconditionally stable scheme of the same accuracy, using the concept used by Chawla ${ }^{24}$, we may re-write (19) into a similar form

$$
\begin{gathered}
\left\{\left[1+\gamma_{1} b\right] \delta_{t}^{2}+\sqrt{a}\left(2 \mu_{t} \delta_{t}\right)-\gamma_{2} P_{x}^{2} \delta_{x}^{2} \delta_{t}^{2}\right\} \phi_{l, m}^{j} \\
+\left\{b-\gamma_{3} P_{y}^{2} \delta_{y}^{2} \delta_{t}^{2}-P_{x}^{2} \delta_{x}^{2}-P_{y}^{2} \delta_{y}^{2}\right\} \phi_{l, m}^{j}
\end{gathered}
$$$$
=k^{2} f_{l, m}^{j} \text {. }
$$

where $\gamma_{1}, \gamma_{2}, \gamma_{3}$ are parameters to be found and added high order parts do not change the accuracy of the scheme.

Let us suppose that, there exists an error $\epsilon_{l, m}^{j}=\Phi_{l, m}^{j}-\phi_{l, m}^{j}$ at each interior nodal point $\left(x_{l}, y_{m}, t_{j}\right)$, the corresponding error equation is given by

$$
\begin{aligned}
&\left\{\left[1+\gamma_{1} b\right]\right. \delta_{t}^{2}+\sqrt{a}\left(2 \mu_{t} \delta_{t}\right)-\gamma_{2} P_{x}^{2} \delta_{x}^{2} \delta_{t}^{2} \epsilon_{l, m}^{j} \\
&+\left\{b-\gamma_{3} P_{y}^{2} \delta_{y}^{2} \delta_{t}^{2}-P_{x}^{2} \delta_{x}^{2}-P_{y}^{2} \delta_{y}^{2}\right\} \epsilon_{l, m}^{j}
\end{aligned}
$$

$=L T E$.

For the stability region, the corresponding characteristic equation can be attained by putting $\epsilon_{l, m}^{j}=\xi^{j} e^{i \theta l} e^{i \beta m}$ in the non-homogeneous part of the error Eq. (21) as

$A_{0} \xi^{2}+B_{0} \xi+C_{0}=0$,

where

$$
\begin{aligned}
& A_{0}=1+\gamma_{1} b+\sqrt{a}+4 \gamma_{2} P_{x}^{2} \sin ^{2} \frac{\theta}{2} \\
& +4 \gamma_{3} P_{y}^{2} \sin ^{2} \frac{\beta}{2}, \\
& \quad B_{0}=4 P_{y}^{2} \sin ^{2} \frac{\beta}{2}-8 \gamma_{2} P_{x}^{2} \sin ^{2} \frac{\theta}{2}-8 \gamma_{3} P_{y}^{2} \sin ^{2} \frac{\beta}{2} \\
& -2-2 \gamma_{1} b+b+4 P_{x}^{2} \sin ^{2} \frac{\theta}{2}, \\
& C_{0}=1+\gamma_{1} b-\sqrt{a}+4 \gamma_{2} P_{x}^{2} \sin ^{2} \frac{\theta}{2} \\
& +4 \gamma_{3} P_{y}^{2} \sin ^{2} \frac{\beta}{2},
\end{aligned}
$$

For stability, the necessary and sufficient conditions for $|\xi|<1$ are that

$A_{0}+B_{0}+C_{0}>0, A_{0}-C_{0}>0$ and $A_{0}-B_{0}+C_{0}>0$.

The condition $A_{0}+B_{0}+C_{0}$

$=b+4\left[P_{x}^{2} \sin ^{2} \frac{\theta}{2}+P_{y}^{2} \sin ^{2} \frac{\beta}{2}\right]>0$

is satisfied for $\alpha_{0}>0, \beta_{0} \geq 0$ and $\forall \theta, \beta$ excluding $\theta=\beta=$ 0 or $2 \pi$ and $\beta_{0}=0$.

We will take care of this case at the end of this section.

The condition $A_{0}-C_{0}=2 \sqrt{a}>0$

is satisfied for all $\alpha_{0}>0, \beta_{0} \geq 0$ and for $\theta, \beta$.

Finally, the condition

$$
\begin{aligned}
& A_{0}-B_{0}+C_{0}=\left(4 \gamma_{1}-1\right) b+4\left(4 \gamma_{2}-1\right) P_{x}^{2} \sin ^{2} \frac{\theta}{2} \\
& +4+4\left(4 \gamma_{3}-1\right) P_{y}^{2} \sin ^{2} \frac{\beta}{2}>0
\end{aligned}
$$


must be satisfied for all $\alpha_{0}>0, \quad \beta_{0} \geq 0$ provided $\gamma_{1} \geq$ $\frac{1}{4}, \gamma_{2} \geq \frac{1}{4}, \gamma_{3} \geq \frac{1}{4}$

When $\theta=\beta=0$ or $2 \pi$ and $\beta_{0}=0$, the characteristic eq. (22) becomes

$(1+\sqrt{a}) \xi^{2}-2 \xi+(1-\sqrt{a})=0$

On solving (29), we get $\xi_{1}=1$ and

$\xi_{2}=(1-\sqrt{a}) /(1+\sqrt{a})=(1-\alpha k) /(1+\alpha k)$.

Here we also find that $|\xi| \leq 1$ and the method (20) is stable.

Thus for $\alpha_{0}>0, \beta_{0} \geq 0, \quad \gamma_{1} \geq \frac{1}{4}, \gamma_{2} \geq \frac{1}{4}, \gamma_{3} \geq \frac{1}{4} \quad$ (i.e. $\gamma_{1}, \gamma_{2}, \gamma_{3}$ are free of $h_{x}, h_{y}$ and $k$ ), the method (20) is stable.

Re-arranging the terms, the method (20) can be written as

$\left\{\left[1+\gamma_{1} b-\gamma_{2} P_{x}^{2} \delta_{x}^{2}\right] \delta_{t}^{2}+\sqrt{a}\left(2 \mu_{t} \delta_{t}\right)\right\}$

$\times\left[1-\gamma_{3} P_{y}^{2} \delta_{y}^{2}\right] \phi_{l, m}^{j}=R_{\phi}$

The added high order extra terms does not influence the accurateness of the method. To assist the numerical reckoning, the method (31) can be written in two-step $\mathrm{ADI}^{22,23}$ form

$\left[1-\gamma_{3} P_{y}^{2} \delta_{y}^{2}\right] \phi_{l, m}^{*}=R_{\phi}$

$\left[1+\gamma_{1} b-\gamma_{2} P_{x}^{2} \delta_{x}^{2}\right] \delta_{t}^{2} \phi_{l, m}^{j}+\sqrt{a}\left(2 \mu_{t} \delta_{t}\right) \phi_{l, m}^{j}$

$=\phi_{l, m}^{*}$

where $\phi_{l, m}^{*}$ is treated as an intermediate boundary value. To solve (32), required intermediate boundary values can be acquired from (33). Both the systems (32) and (33) are tridiagonal systems and thus, can be solved employing a tridiagonal solver.

\section{Computational Results}

We have solved several standard problems arising from physics and engineering using the method (16). The analytical solutions are known in each example. We determined the right-hand side homogenous function $f(x, y, t)$, initial \& boundary conditions using the analytical solution as a test process. The Gauss elimination (tridiagonal solver) method can be employed for solving the linear difference equation and the Newton-Raphson method for non-linear difference equations ${ }^{20,21}$.We use MATLAB codes to perform all the numerical computations.

The presented methods (16) and (20) for second-order hyperbolic equations are three-level implicit methods. To commence the estimation, it is compulsory to calculate the numerical solution of $\phi$ of the desired accuracy at $t=k$.
As it is given the values of $\phi$ and $\phi_{t}$ at $t=0$ explicitly, we can determine the values of subsequent tangential derivatives of $\phi$ and $\phi_{t}$ at $t=0$, which implies that at $t=0$, the values of $\phi, \phi_{x}, \phi_{y}, \phi_{x x}, \phi_{y y} \ldots \ldots$, $\phi_{t}, \phi_{t x}, \phi_{t y}, \phi_{t x x}, \phi_{t y y} \ldots$ etc are known.

Using Taylor's expansion, an approximation at first time level is given by

$$
\Phi_{l, m}^{1}=\Phi_{l, m}^{0}+k\left(\Phi_{t}\right)_{l, m}^{0}+\frac{k^{2}}{2}\left(\Phi_{t t}\right)_{l, m}^{0}+O\left(k^{3}\right)
$$

From Eq. (1), we have

$$
\begin{aligned}
& \left(\Phi_{t t}\right)_{l, m}^{0}=\left[A(x, y, t, \Phi) \Phi_{x x}+B(x, y, t, \Phi) \Phi_{y y}\right. \\
& \left.+R\left(x, y, t, \Phi, \Phi_{x}, \Phi_{y}, \Phi_{t}\right)\right]_{l, m}^{0}
\end{aligned}
$$

From the equation (35), we can estimate the value of $\Phi_{t t}$ at $t=0$ using the initial values of $\Phi$ and its derivatives and automatically the value of $\Phi$ at first time level i.e. at $t=k$ can be obtained from the equation (34) to the desired accuracy.

Example 1: We solved the linear telegraphic equation (18) in the solution domain $0<x<1,0<y<\sqrt{2}, t>0$. The analytical result is given by $\phi(x, y, t)=\exp (-2 t) \sinh x \sinh y$. The maximum absolute errors (MAEs) are reported in Table 1 at $t=5$ for a pre-set value of $\sigma_{x}=k(N+1)^{2}=3.2$ and different values of $\alpha_{0}, \beta_{0}, \gamma_{1}, \gamma_{2}, \gamma_{3}$. Figures 1 display the analytical and numerical solution at $t=5$ for $\alpha_{0}=10, \beta_{0}=5, \gamma_{1}=$ $0.5, \gamma_{2}=\gamma_{3}=1.0$ and $N=M=31$.

Example 2: (Wave equation in polar coordinates

$\phi_{t t}=\phi_{r r}+\phi_{z z}+\frac{1}{r} \phi_{r}+f(r, z, t)$,

$0<r<1,0<z<1 / \sqrt{2}, t>0$

The above equation (36) represents the $2 \mathrm{D}$ wave equation in cylindrical polar coordinates. The analytical solution is given by $\phi(r, z, t)=\exp (-2 t) \cosh r \cosh z$. The MAEs are tabulated in Table 2 at $t=1.0$ and $t=2.0$ for a pre-set value of $\sigma_{x}=k(N+1)^{2}=0.8$. The analytical and numerical solution curves are plotted in Figures 2 at $t=1.0$ for $N=M=31$.

Example 3: (Van-der Pol type nonlinear wave equation)

$\phi_{t t}=\phi_{x x}+\phi_{y y}+\gamma\left(\phi^{2}-1\right) \phi_{t}+f(x, y, t), \quad 0<x<$ $1,0<y<\sqrt{2}, t>0$

The analytical solution is given by $\phi(x, y, t)=$ $\exp (-\gamma t) \sin (\pi x) \sin (\pi y)$. The MAEs at $t=1$ are reported in Table 3 for $\gamma=1,2,3$ with the fixed value of $\sigma_{x}=$ $k(N+1)^{2}=0.8$. Figures 3 the analytical and numerical solution curves at $t=1.0$ for $\gamma=1$ and $N=M=31$. 
Table 1. Example 1: The MAEs at $t=5, k=3.2(N+1)^{-2}$

\begin{tabular}{|l|c|c|c|c|c|}
\hline & $\alpha=10, \beta=5$, & $\alpha=20, \beta=10$, & $\alpha=40, \beta=4$, & $\alpha=50, \beta=5$, & $\alpha=10, \beta=0$, \\
$N=M$ & $\gamma_{1}=0.5$, & $\gamma_{1}=1.0$, & $\gamma_{1}=10.0$, & $\gamma_{1}=0.25$, & $\gamma_{2}=0.50$, \\
& $\gamma_{2}=1.0$, & $\gamma_{2}=1.0$, & $\gamma_{2}=20.0$, & $\gamma_{3}=5.0$, & $\gamma_{2}=5.0$, \\
\hline 15 & $\gamma_{3}=1.0$ & $\gamma_{3}=1.0$ & $\gamma_{3}=30.0$ & $8.9809 \mathrm{e}-06$ & $1.5871 \mathrm{e}-05$ \\
\hline 31 & $1.1480 \mathrm{e}-05$ & $1.1944 \mathrm{e}-05$ & $1.3203 \mathrm{e}-05$ & $2.2841 \mathrm{e}-06$ & $3.8909 \mathrm{e}-06$ \\
\hline 63 & $3.0656 \mathrm{e}-06$ & $2.8367 \mathrm{e}-06$ & $3.3496 \mathrm{e}-06$ & $5.6474 \mathrm{e}-07$ & $9.1982 \mathrm{e}-07$ \\
\hline
\end{tabular}
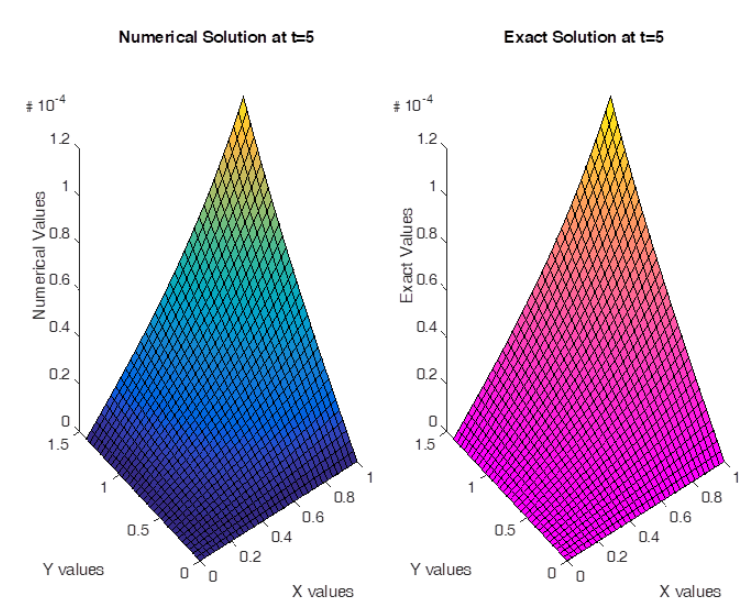

Fig. 1. The graph of numerical vs. exact solution of example 1 at $t=5, k=3.2(N+1)^{-2}, N=M=31$.

Table 2.Example 2: The MAEs for $k=0.8(N+1)^{-2}$

\begin{tabular}{|l|l|l|}
\hline \multirow{2}{*}{$N=M$} & Proposed method & \multicolumn{1}{c|}{$t=2.0$} \\
\cline { 2 - 3 } & \multicolumn{1}{|c|}{$t=1.0$} & $1.9533 \mathrm{e}-05$ \\
\hline 15 & $5.1323 \mathrm{e}-05$ & $4.9905 \mathrm{e}-06$ \\
\hline 31 & $1.2807 \mathrm{e}-05$ & $1.3757 \mathrm{e}-06$ \\
\hline 63 & $3.1750 \mathrm{e}-06$ & \\
\hline
\end{tabular}

Example 4: (Dissipative nonlinear wave equation)

$\phi_{t t}=\phi_{x x}+\phi_{y y}+-2 \phi \phi_{t}+f(x, y, t)$,

$0<x<1,0<y<1 / \sqrt{2}, t>0$

The analytical solution is given by $\phi(x, y, t)=$ $\sin (\pi x) \sin (\pi y) \cosh t$. For a fixed value of $\sigma_{x}=$ $k(N+1)^{2}=0.8$, the MAEs are tabulated in Table 4 at $t=1$ and 2. Figures 4 display the analytical and numerical solution curves at $t=1$ for $N=M=31$.

Example 5: (Quasi-linear hyperbolic equation)

$\phi_{t t}=\left(1+\phi^{2}\right)\left(\phi_{x x}+\phi_{y y}\right)+\gamma \phi\left(\phi_{x}+\phi_{y}+\phi_{t}\right)$

$+f(x, y, t), 0<x<1,0<y<\sqrt{3}, t>0$

The analytical solution is given by $\phi(x, y, t)=$ $\exp (-2 t) \sin (\pi x) \sin (\pi y)$. The MAEs at $t=1$ and 2 are reported in Table 5 for $\gamma=1,2$, and 3 with the fixed value of $\sigma_{x}=k(N+1)^{2}=0.8$. Figures 5 display the analytical and numerical solution curves at $t=1$ for $\gamma=1$ and $N=M=31$.
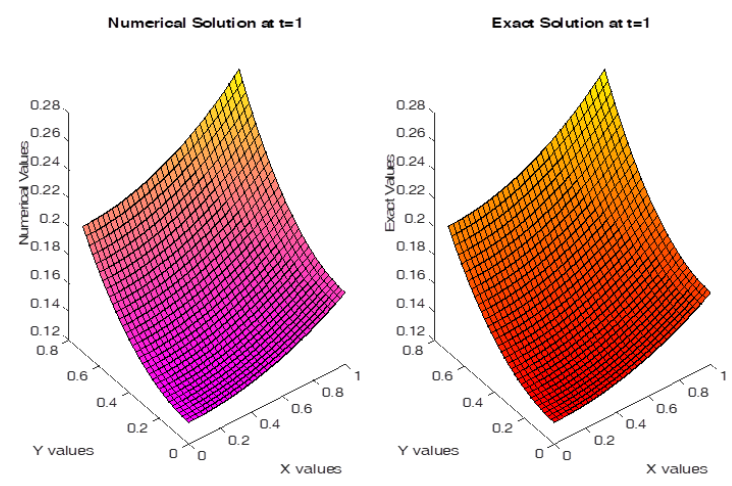

Fig. 2. The graph of numerical vs. exact solution of example 2 at $t=1, k=0.8(N+1)^{-2}, N=M=31$.

Table 3. Example 3: The MAEs for $k=0.8(N+1)^{-2}$

\begin{tabular}{|l|l|l|c|}
\hline \multirow{2}{*}{$N=M$} & \multicolumn{3}{|c|}{ Proposed method } \\
\cline { 2 - 4 } & $\gamma=1.0$ & $\gamma=2.0$ & $\gamma=3.0$ \\
\hline 15 & $3.2872 \mathrm{e}-03$ & $1.6711 \mathrm{e}-03$ & $9.7153 \mathrm{e}-04$ \\
\hline 31 & $8.1387 \mathrm{e}-04$ & $4.1590 \mathrm{e}-04$ & $2.4185 \mathrm{e}-04$ \\
\hline 63 & $2.0339 \mathrm{e}-04$ & $1.0404 \mathrm{e}-04$ & $6.0481 \mathrm{e}-05$ \\
\hline
\end{tabular}

Table 4. Example 4: The MAEs for $k=0.8(N+1)^{-2}$

\begin{tabular}{|l|l|l|}
\hline \multirow{2}{*}{$N=M$} & \multicolumn{1}{|c|}{$t=1.0$} & \multicolumn{1}{c|}{$t=2.0$} \\
\cline { 2 - 3 } & $1.8779 \mathrm{e}-03$ & $4.5233 \mathrm{e}-03$ \\
\hline 15 & $4.7013 \mathrm{e}-04$ & $1.1309 \mathrm{e}-03$ \\
\hline 31 & $1.1743 \mathrm{e}-04$ & $2.8271 \mathrm{e}-04$ \\
\hline 63 & Exact Solution at $\mathrm{t}=1$ \\
\hline
\end{tabular}

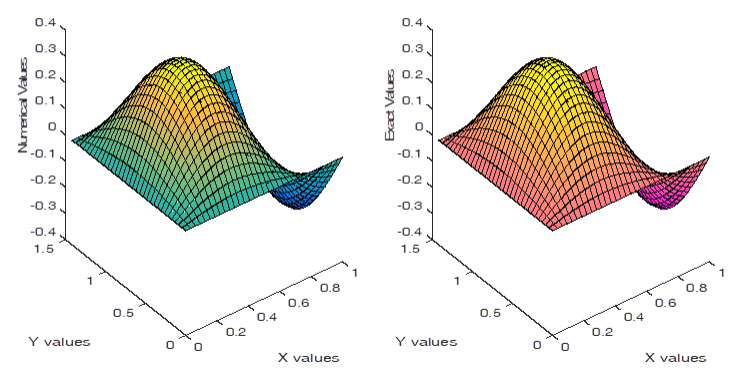

Fig. 3. The graph of numerical vs. exact solution of example 3 at $t=1, \gamma=1, k=0.8(N+1)^{-2}, N=M=31$. 

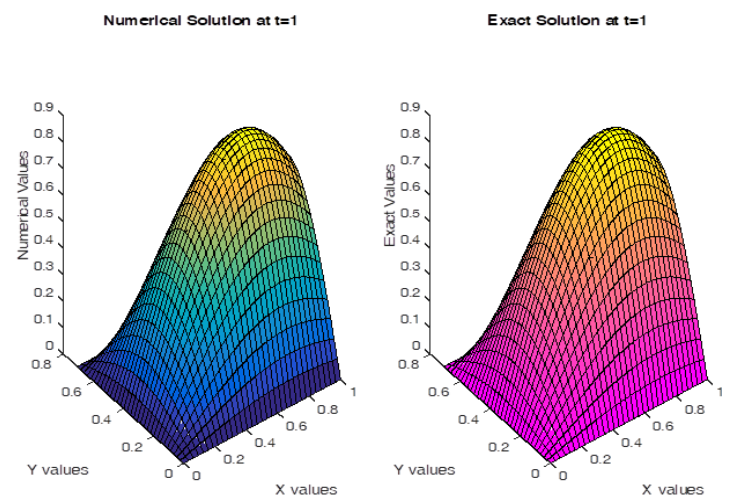

Fig. 4. The graph of numerical vs. exact solution of example 4 at $t=1, k=0.8(N+1)^{-2}, N=M=31$.

Table 5. Example 5: The MAEs for $k=0.8(N+1)^{-2}$

\begin{tabular}{|l|l|l|l|}
\hline \multirow{2}{*}{$N=M$} & \multicolumn{3}{|l|}{ Proposed method } \\
\cline { 2 - 4 } & $\gamma=1.0$ & $\gamma=2.0$ & $\gamma=3.0$ \\
\hline 15 & $4.1836 \mathrm{e}-03$ & $4.0046 \mathrm{e}-03$ & $4.2300 \mathrm{e}-03$ \\
\hline 31 & $1.0568 \mathrm{e}-03$ & $1.0302 \mathrm{e}-03$ & $1.1332 \mathrm{e}-03$ \\
\hline 63 & $2.6491 \mathrm{e}-04$ & $2.5808 \mathrm{e}-04$ & $2.8801 \mathrm{e}-04$ \\
\hline
\end{tabular}

Table 6. Example 6: The MAEs for $k=0.8(N+1)^{-2}$

\begin{tabular}{|l|l|l|l|}
\hline \multirow{2}{*}{$N=M$} & \multicolumn{3}{|c|}{ Proposed method } \\
\cline { 2 - 4 } & $\gamma=1.0$ & $\gamma=2.0$ & $\gamma=3.0$ \\
\hline 15 & $1.1672 \mathrm{e}-02$ & $5.2959 \mathrm{e}-02$ & $3.2867 \mathrm{e}-01$ \\
\hline 31 & $2.9263 \mathrm{e}-03$ & $1.2989 \mathrm{e}-02$ & $7.6211 \mathrm{e}-02$ \\
\hline 63 & $7.3140 \mathrm{e}-04$ & $3.2318 \mathrm{e}-03$ & $1.8655 \mathrm{e}-02$ \\
\hline
\end{tabular}

Numerical Solution at $\mathrm{t}=1$

Exact Solution at $t=1$
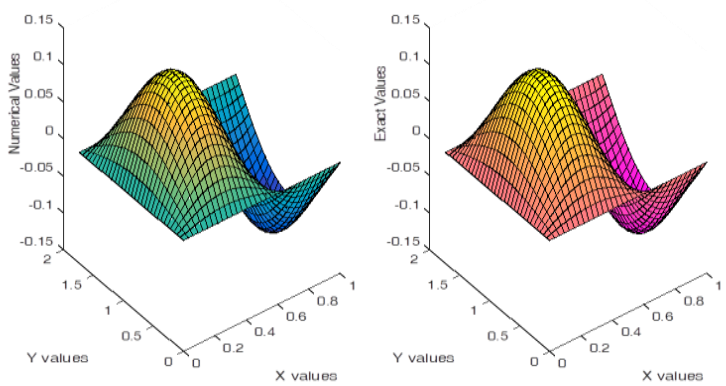

Fig. 5. The graph of numerical vs. exact solution of Example 5 at $t=1, \gamma=1, k=0.8(N+1)^{-2}, N=M=31$.
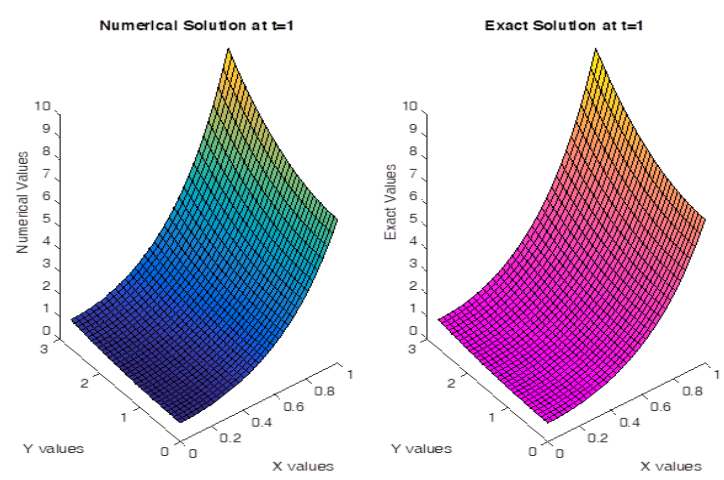

Fig. 6. The graph of numerical vs. exact solution of Example 6 at $t=1, k=0.8(N+1)^{-2}, N=M=31$.
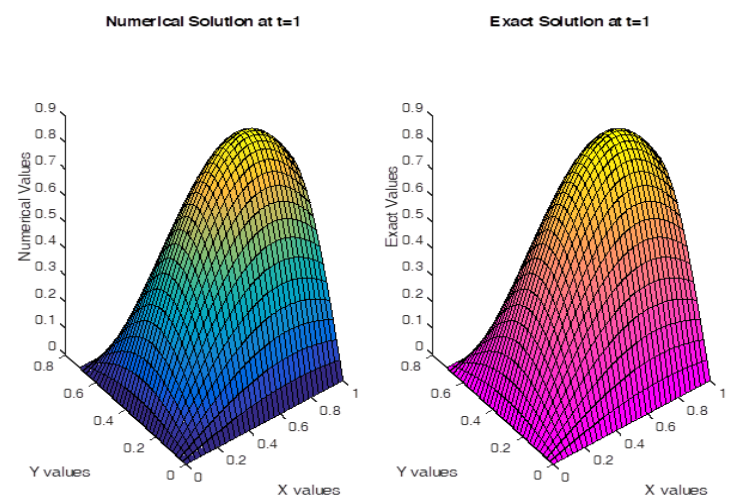

Fig. 7. The graph of numerical vs. exact solution of Example 7 at $t=1, k=0.8(N+1)^{-2}, N=M=31$.

Example 6: (Nonlinear wave equation with variable coefficients)

$$
\begin{aligned}
& \phi_{t t}=\left(1+x^{2}\right) \phi_{x x}+\left(1+y^{2}\right) \phi_{y y}+\gamma \phi\left(\phi_{x}+\phi_{y}\right. \\
& \left.+\phi_{t}\right)+f(x, y, t), 0<x<1,0<y<e, t>0
\end{aligned}
$$

The analytical solution is given by $\phi(x, y, t)=$ $\sin t \cosh x \cosh y$. The MAEs at $t=1$ and 2 are reported in Table 6 for $\gamma=1,2$, and 3 with the fixed value of $\sigma_{x}=$ $k(N+1)^{2}=0.8$. Figures 6 represent the analytical and numerical solution curves at $t=1$ for $\gamma=1$ and $N=M=$ 31.

Example 7: (Nonlinear Ohm's law)

$$
\begin{aligned}
& \phi_{t t}+A(\phi) \phi_{t}=\frac{\partial}{\partial x}\left[g(\phi) \phi_{x}\right]+\frac{\partial}{\partial y}\left[g(\phi) \phi_{y}\right] \\
& +f(x, y, t), 0<x<1,0<y<1 / \sqrt{2}, t>0
\end{aligned}
$$

For $g(\phi)=$ constant, the equation of the type (41) encountered in the theory of electric field and nonlinear Ohm's law, $\phi$ is the electric field strength ${ }^{25}$. The analytical solution is given by $\phi(x, y, t)=\sin (\pi x) \sin (\pi y) \sin t$. The MAEs are tabulated in Table 7 at $t=1$ and $\mathrm{t}=2$ for $A(\phi)=\phi^{2}, g(\phi)=1$ and for a fixed value of $\sigma_{x}=$

\begin{tabular}{|c|c|c|}
\hline \multirow{2}{*}{$N=M$} & \multicolumn{2}{|c|}{ Proposed method } \\
\hline & $t=1.0$ & $t=2.0$ \\
\hline 15 & $1.5606 \mathrm{e}-03$ & $1.7889 \mathrm{e}-03$ \\
\hline 31 & $3.8994 \mathrm{e}-04$ & $4.4670 \mathrm{e}-04$ \\
\hline 63 & $9.7555 e-05$ & $1.1174 \mathrm{e}-04$ \\
\hline
\end{tabular}
$k(N+1)^{2}=0.8$. The analytical and numerical solution curves are plotted in Figures 7 at $t=1$ for $N=M=31$.

Table 7. Example 7: The MAEs fork $=0.8(N+1)^{-2}$

The proposed method (16) is of $O\left(k^{2}+h_{x}^{2}+h_{y}^{2}\right)$. Since we have used unequal mesh, that is, $h_{x} \propto h_{y}$, the LTE given in (16) reduces to $O\left(k^{2}+h_{x}^{2}\right)$. Further, when $k \propto$ $h_{x}$, the proposed method becomes of $O\left(h_{x}^{2}\right)$, that is, order two in space. In order to show the second order convergence, we have chosen $k=3.2 h_{x}^{2}$ in example 1 and $k=0.8 h_{x}^{2}$ in other examples. We have estimated the order of convergence using the formula 


$$
\frac{\log \left(e_{h_{x 1}}\right)-\log \left(e_{h_{x 2}}\right)}{\log \left(h_{x 1}\right)-\log \left(h_{x 2}\right)}
$$

where maximum absolute errors are $\mathrm{e}_{h_{x 1}}$ and $\mathrm{e}_{h_{x 2}}$ for two different uniform mesh sizes $h_{x 1}=\frac{a}{\left(1+L_{x 1}\right)}$ and $h_{x 2}=$ $\frac{a}{\left(1+L_{x 2}\right)}$ respectively. To calculate the order of convergence of the suggested method, MAEs for the last two values of $h_{x}$, i.e., $h_{x 1}=1 / 32$ and $h_{x 2}=1 / 64$ (for linear problems) and $h_{x 1}=1 / 16$ and $h_{x 2}=1 / 32$ (for nonlinear and quasilinear problems) have been considered, and corresponding results are presented in Table 8.

It has been verified that there is a reduction in the values of the MAEs by about 1/4, when the value of $h_{x}$ is reduced by $\frac{1}{2}$. Clearly, the experimental results are consistent and endorse the second-order convergence of the suggested method. Further, it is not possible to plot the graph in fourdimensions $(u, t, x, y)$. For fixed values of $t$, we have plotted the graphs for both numerical and exact solutions in threedimensions. The numerical and exact solution graphs are nearly identical for a fixed value of $t$ and $z$.

\section{Conclusion}

Available developednumerical schemes quoted in the references for the solution of $2^{\text {nd }}$ order quasi-linear hyperbolic PDEs are useful for computation on a uniform mesh with equal mesh sizes. In the present paper, we have considered an irrational domain $\Omega \equiv\{(x, y, t): 0<x<$ $a, 0<y<b, t>0\}$, with unequal mesh sizes andestablished a novel stable numerical scheme of $O\left(k^{2}+\right.$ $h_{x}^{2}+h_{y}^{2}$ ) so that many real-world 2D engineering problems can be solved. The suggested scheme is appropriate to solve wave equations in polar/cylindrical coordinates. The developed technique is revealed to be unconditionally stable when applied to the telegraphic equation, and the stability criterion is established. Further, our method is not applicable on variable mesh and hyperbolic equations with mixed derivative terms. We have solved some noteworthy problems both linear and nonlinear HPDEs to justify the usefulness of the suggested method. The proposed technique can be extended to develop a scheme of $O\left(k^{2}+h_{x}^{4}+h_{y}^{4}\right)$ to solve 2D quasi-linear HPDEs on an irrational domain.

\section{References}

1. Li, W.D., Z. Z. Sun and L. Zhao, 2007. An analysis for a high order difference scheme for numerical solution to $u_{t t}=$ $A(x, t) u_{x x}+f\left(x, t, u, u_{x}, u_{t}\right)$. Numerical Methods for Partial Differential Equations, 23, 484-498.

2. Greenspan, D., 1968. Approximate solution of initial boundary wave equation problems by boundary values techniques. Communication of the ACM, 11, $760-763$.

3. Ciment, M. and S.H. Leventhal, 1975. Higher order compact implicit schemes for the wave equation. Mathematics of Computation, 29, 985 - 994.

4. Ciment, M. and S.H. Leventhal, 1978. A note on the operator compact implicit method for the wave equation. Mathematics of Computation, 32, 143 - 147.

5. Twizell, E.H., 1979. An explicit difference method for the wave equation with extended stability range. BIT, 19, $378-$ 383.

6. Mohanty, R.K., M.K. Jain and K. George, 1996. High order difference schemes for the system of two space second order nonlinear hyperbolic equations with variable coefficients. Journal of Computational and Applied Mathematics, 70, 231-243.

7. Mohanty, R.K., U. Arora and M.K. Jain, 2001. Linear stability analysis and fourth-order approximations at first level for the two space dimensional mildly quasi-linear hyperbolic equations. Numerical Methods for Partial Differential Equations, 17, 607-618.

Table 8. Order of the convergence

\begin{tabular}{|c|c|c|}
\hline Example & Parameters & Order of the method \\
\hline 01 & $\begin{array}{cc}\alpha=10, \beta=5.0, \gamma_{1}=0.5, \gamma_{2}=1.0, \gamma_{2}=1.0 & \text { at } t=5 \\
\alpha=20, \beta=10, \gamma_{1}=1.0, \gamma_{2}=1.0, \gamma_{2}=1.0 & \text { at } t=5 \\
\alpha=40, \beta=4.0, \gamma_{1}=10, \gamma_{2}=20, \gamma_{2}=30 & \text { at } t=5 \\
\alpha=50, \beta=5.0, \gamma_{1}=0.25, \gamma_{2}=0.50, \gamma_{2}=0.75 & \text { at } t=5 \\
\alpha=10, \beta=0.0, \gamma_{1}=5.0, \gamma_{2}=5.0, \gamma_{2}=5.0 & \text { at } t=5\end{array}$ & $\begin{array}{l}2.0923 \\
2.0037 \\
2.0268 \\
2.0160 \\
2.0807\end{array}$ \\
\hline 02 & $\begin{array}{l}\text { at } t=1.0 \\
\text { at } t=2.0\end{array}$ & $\begin{array}{l}2.0027 \\
1.9687 \\
\end{array}$ \\
\hline 03 & $\begin{array}{l}\gamma=1.0 \text { at } t=1.0 \\
\gamma=2.0 \text { at } t=1.0 \\
\gamma=3.0 \text { at } t=1.0\end{array}$ & $\begin{array}{l}2.0140 \\
2.0065 \\
2.0061 \\
\end{array}$ \\
\hline 04 & $\begin{array}{l}\text { at } t=1.0 \\
\text { at } t=2.0\end{array}$ & $\begin{array}{l}1.9980 \\
1.9999 \\
\end{array}$ \\
\hline 05 & $\begin{array}{l}\gamma=1.0 \text { at } t=1.0 \\
\gamma=2.0 \text { at } t=1.0 \\
\gamma=3.0 \text { at } t=1.0\end{array}$ & $\begin{array}{l}1.9850 \\
1.9587 \\
1.9003 \\
\end{array}$ \\
\hline 06 & $\begin{array}{l}\gamma=1.0 \text { at } t=1.0 \\
\gamma=2.0 \text { at } t=1.0 \\
\gamma=3.0 \text { at } t=1.0\end{array}$ & $\begin{array}{l}1.9959 \\
2.0276 \\
2.0186 \\
\end{array}$ \\
\hline 07 & $\begin{array}{l}\text { at } t=1.0 \\
\text { at } t=2.0\end{array}$ & $\begin{array}{l}2.0008 \\
2.0017 \\
\end{array}$ \\
\hline
\end{tabular}


8. Mohanty, R.K. and M.K. Jain, 2001. An unconditionally stable alternating direction implicit scheme for the two space dimensional linear hyperbolic equation, Numerical Methods for Partial Differential Equations, 22, 983-993.

9. Mohanty, R.K., 2004. An operator splitting method for an unconditionally stable difference scheme for a linear hyperbolic equation with variable coefficients in two space dimensions. Applied Mathematics and Computation, 152, 799-806.

10. Mohanty, R.K., 2007. Stability interval for explicit difference schemes for multi-dimensional second order hyperbolic equations with significant first order space derivative terms. Applied Mathematics and Computations, 190, 1683-1690.

11. Dehghan, M. and A. Shokri, 2008. A numerical method for solving the hyperbolic telegraph equation. Numerical Methods for Partial Differential Equations, 24, 1080-1093.

12. Dehghan, M. and A. Mohebbi, 2009. High order implicit collocation method for the solution of two dimensional linear hyperbolic equation. Numerical Methods for Partial Differential Equations, 25, 232-243.

13. Dehghan, M. and A. Shokri, 2009. A meshless method for numerical solution of a linear hyperbolic equation with variable coefficients in two space dimensions. Numerical Methods for Partial Differential Equations, 25, 494-506.

14. Mohanty, R.K., 2009. New unconditionally stable difference schemes for the solution of multi-dimensional telegraphic equations. International Journal of Computer Mathematics, 86, 2061-2071.

15. Liu, J. and K. Tang, 2010. A new unconditionally stable ADI compact scheme for the two-space dimensional linear hyperbolic equation. International Journal of Computer Mathematics, 87, 2259-2267.

16. Karaa, S., 2010. Unconditionally stable ADI scheme of higher-order for linear hyperbolic equations. International Journal of Computer Mathematics, 87, 3030-3038.
17. Mohanty, R.K., 2014. New high accuracy super stable alternating direction implicit methods for two and three dimensional hyperbolic damped wave equations. Results in Physics, 4, 156-163.

18. Priyadarshini, I. and R.K. Mohanty, 2021. High resolution compact numerical method for the System of 2D quasi-linear elliptic boundary value problems and the solution of normal derivatives on an irrational domain with engineering applications. Engineering with Computers, DOI: 10.1007/s00366-020-01150-4.

19. Priyadarshini, I. and R.K. Mohanty, 2021. High resolution half-step compact numerical approximation for 2D quasilinear elliptic equations in vector form and the estimates of normal derivatives on an irrational domain, Soft Computing, DOI: $10.1007 / \mathrm{s} 00500-020-05505-3$.

20. Kelly, C.T., 1995. Iterative Methods for Linear and NonLinear Equations, SIAM Publications, Philadelphia, USA.

21. Hageman, L.A. and D.M. Young, 2004. Applied Iterative Methods, Dover Publication, New York.

22. Lees, M., 1962. Alternating direction methods for hyperbolic differential equations, Journal of the Society for Industrial and Applied Mathematics, 10, 610-616.

23. Gourlay, A.R. and A.R. Mitchell, 1969. A classification of split difference methods for hyperbolic equations in several space dimensions, SIAM Journal on Numerical Analysis, 6 , $62-71$.

24. Chawla, M.M., 1985. Superstable two-step methods for the numerical integration of general second order initial value problem,Journal of Computational and Applied Mathematics, 12, 217-220.

25. Polyanin, A.D. and V.F. Zaitsev, 2003. Nonlinear partial differential equations, Chapman and Hall/CRC, New York. 